\title{
Selective cerebral revascularization as an adjunct in the treatment of giant anterior circulation aneurysms
}

\author{
Brian A. O’Shaughnessy, M.D., Sean A. Salehi, M.D., Stefan A. Mindea, M.D., \\ AND H. HunT BATJER, M.D.
}

Department of Neurological Surgery, The Feinberg School of Medicine and McGaw Medical Center, Northwestern University, Chicago, Illinois

\begin{abstract}
Cerebral revascularization, an indispensable component of neurovascular surgery, has been performed in the treatment of cranial base tumors, complex cerebral aneurysms, and occlusive cerebrovascular disease. The goal of a revascularization procedure is to augment blood flow distally. It can therefore be used as an adjunctive measure in the treatment of complex neurosurgical disease processes that require parent artery sacrifice for definitive treatment. In the treatment of giant anterior circulation aneurysms, for instance, a cerebral revascularization procedure may be considered in patients in whom the collateral circulation is marginal and in whom lesions may be treated either using a Hunterian-based strategy or clip-assisted reconstruction requiring a prolonged period of temporary occlusion. To date, there is no entirely effective method known to produce long-term tolerance to carotid artery (CA) sacrifice and, largely for that reason, some neurovascular surgeons advocate universal revascularization. The authors of this report, however, prefer to perform revascularization only in the limited subset of patients in whom preoperative assessment has revealed risk factors for cerebral ischemia due to hypoperfusion. In this paper, the authors introduce their protocol for assessing cerebrovascular reserve capacity, indications for cerebral revascularization in the treatment of complex anterior circulation aneurysms, and discuss their rationale for choosing to practice selective, rather than universal, revascularization.
\end{abstract}

\section{KEY WORDS - revascularization - giant aneurysm • extracranial-intracranial bypass • balloon occlusion - cerebrovascular reserve capacity • Hunterian}

The ideal treatment of a giant intracranial aneurysm is definitive exclusion from the circulation by performing microneurosurgical clip-assisted reconstruction in which all afferent and efferent arterial branches in the aneurysm segment are preserved. Unfortunately, because these lesions very frequently involve both the parent artery and critical neighboring branches, reconstruction is rarely a straightforward task. In fact, for most giant aneurysms, clip reconstruction requires a prolonged period of temporary occlusion to obtain optimal results. In some cases, usually because of inaccessibility or an unfavorable neck configuration, clip-assisted reconstruction is not feasible; the giant aneurysm must then be treated using a Hunterian strategy. In his considerable experience treating 174 patients who harbored giant intracranial aneurysms, Drake ${ }^{10}$ reported that $62 \%$ of the lesions were deemed unamenable to placement of a clip; his data underscored the difficulty

Abbreviations used in this paper: $\mathrm{BTO}=$ balloon test occlusion; $\mathrm{CA}=$ carotid artery; $\mathrm{CBF}=$ cerebral blood flow; $\mathrm{CCA}=$ contralat eral CA; $\mathrm{CRT}=$ cerebrovascular reserve test; $\mathrm{CT}=$ computerized tomography; EC-IC = extracranial-intracranial; ECA = external $\mathrm{CA} ; \mathrm{EEG}=$ electroencephalography; ICA = internal CA; $\mathrm{MCA}=$ middle cerebral artery; $\mathrm{MR}=$ magnetic resonance; $\mathrm{SAH}=$ subarachnoid hemorrhage; SPECT $=$ single-photon emission CT. of direct approaches in the management of these complex pathological entities, even in the best of hands.

It is widely accepted that a subset of patients who are treated with a Hunterian occlusion-based strategy are at risk of suffering clinically significant ipsilateral cerebral hypoperfusion. The same holds true for patients requiring prolonged temporary occlusion for clip reconstruction of the aneurysmal segment. Some neurovascular surgeons have performed prophylactic revascularization in all patients harboring complex aneurysms, ${ }^{28,41}$ whereas others ${ }^{10,11,18,22,25,40,50}$ have implemented a selective cerebral revascularization paradigm based on extensive data derived from preoperative testing of cerebrovascular reserve capacity. In the latter of these two schemes, which is our our institutional practice, revascularization is considered to be an adjunctive measure only in patients in whom impairment of cerebrovascular reserve is demonstrated. Moreover, based on both their angiographically depicted neurovascular anatomy and results of CRT, the most appropriate bypass conduit is then selected. In this review, which is based on the neurovascular practice of the senior author (H.H.B.), we describe our approach to evaluation and surgical management of patients with giant anterior circulation aneurysms with a particular focus on our usage of adjunctive cerebral revascularization. We describe the 
management of a patient with a giant supraclinoid CA aneurysm to illustrate further our general principles of practice. Finally, our rationale for advocating selective revascularization is discussed.

\section{SELECTIVE CEREBRAL REVASCULARIZATION}

\section{Initial Neuroradiological Evaluation}

In all patients who will undergo surgical treatment of a giant intracranial aneurysm, the following neurodiagnostic studies, at the very least, have been performed: noncontrast head CT scanning, diffusion-weighted MR imaging, and four-vessel cerebral angiography. It has become our practice to obtain a three-dimensional CT angiogram in every patient with a giant aneurysm in whom some form of surgical treatment is planned. Computerized tomography angiography plays a complementary role to conventional cerebral angiography in the evaluation of aneurysms, primarily by allowing the easy visualization intraaneurysm and neck thrombosis or calcification, as well as depicting the relationship of the lesion to neighboring arteries and important cranial base structures such as the clinoid process. Diffusion-weighted MR imaging is an extremely sensitive modality by which to detect subtle ischemic changes that may predict diminished tolerance to extended arterial occlusion.

\section{Cerebrovascular Reserve Testing}

Assessment of cerebrovascular reserve, which is composed of four principal elements-neuroclinical, hemodynamic, neurophysiological, and provocative-is used in an effort to predict tolerance to parent artery sacrifice. The cornerstone of the CRT is the BTO, which has been widely described in the literature ${ }^{7,13,14,17,31,33,34,37,43,44,47,48}$ as well as in reviews ${ }^{5,23}$ and involves monitoring the awake patient in whom an artery is temporarily occluded by using nondetachable endovascular balloons. Cerebrovascular reserve testing is routinely performed in all our patients with unruptured giant intracranial aneurysms prior to any surgical treatment. In general, however, CRT should be performed in patients harboring aneurysms for which a deconstructive strategy is anticipated or, based on the assessment of an experienced neurovascular surgeon, a prolonged period of temporary occlusion will be required for clip-assisted reconstruction. After the CRT, the case is then stratified based on the outcome. The CRT results not only allow us to predict tolerance to parent artery sacrifice with a high degree of certainty, but they also enable us to select the most appropriate type of bypass conduit should revascularization be necessary as an adjunctive measure.

Investigators in the Cooperative Study of Intracranial Aneurysms and Subarachnoid Hemorrhage ${ }^{36}$ reported that the rates of ischemic cerebral-related complications attributed to abrupt CA occlusion were 49 and $28 \%$ for ICA and CCA sacrifice, respectively. This wide discrepancy in values has been attributed to several factors. First, in cases requiring CCA sacrifice, because the ICA frequently remains fully patent, clinically significant thromboembolic phenomena affecting the ipsilateral hemisphere are less likely to occur. ${ }^{19,46}$ Usually retrograde flow through the ipsilateral ECA allows substantial orthograde ICA flow, thereby limiting ischemic sequelae. Moreover, the dysautoregulatory state associated with SAH limits the capacity of regional cerebral circulation vasodilation to maintain adequate perfusion during $\mathrm{CA}$ occlusion. In cases in which the BTO is clinically tolerated-the neuroclinical arm of the CRT-the rate of cerebral ischemia following CA sacrifice decreases to somewhere between 5 and $20 \%$. $^{7,17}$ With the goal of improving the predictive ability of the BTO, quantitative hemodynamic assessment in the form of radionuclide SPECT imaging-either using ${ }^{99 \mathrm{~m}} \mathrm{Tc} \mathrm{HMPAO}$ or ${ }^{133} \mathrm{Xe}$ - has been added to the CRT protocol. ${ }^{7,14,26}$ Augmentation of the CRT schema with these CBF studies has met with some, but not overwhelming, success at improving our ability to predict tolerance to parent artery sacrifice. In a study that combined neuroclinical assessment during BTO and nonquantitative ${ }^{99 \mathrm{~m}} \mathrm{Tc}$ HMPAO SPECT, Peterman, et al. ${ }^{37}$ found that two of 17 patients in whom BTO was tolerated asymmetrical cerebral perfusion was demonstrated on radionuclide $\mathrm{CBF}$ studies. One of these two patients is currently undergoing follow-up study, whereas significant EEG slowing was demonstrated in the other during temporary arterial occlusion. Conversely, one of the 15 patients in their series in whom BTO was tolerated and symmetrical perfusion was revealed on ${ }^{99 \mathrm{~m} T c-H M P A O-S P E C T ~ i m a g i n g ~ e x p e r i e n c e d ~}$ a watershed infarction during a period of intraoperative hypotension. Results obtained in this study, and others just like it, ${ }^{13,33}$ clearly underscore the multifactorial complexity of arterial blood flow to the brain and its potential vulnerability to a variation in systemic hemodynamics, particularly hypotension, volume depletion, and arrhythmias. As a consequence of these factors, the CRT now commonly incorporates periods of hypotension-provocative testing - that are iatrogenically induced using either sodium nitroprusside or labetalol to achieve a blood pressure roughly two thirds of mean arterial pressure. In their seminal study on this topic, Standard and colleagues ${ }^{43}$ retrospectively reviewed 47 cases of BTO performed at the State University of New York at Buffalo. Of the 19 patients who underwent iatrogenic CA sacrifice after a clinically tolerated BTO involving hypotensive challenge, only one experienced a delayed ischemic insult-a minor cerebral infarction in the distribution of the distal posterior division of the MCA, certainly due to thromboembolism. Of interest, the surgical procedure in this patient involved Hunterian ligation, which is thought to be associated with a higher rate of thromboembolic complications than endovascular CA occlusion due to, among other things, formation of thrombus in CA "dead space."

As previously mentioned, our institutional protocol for CRT assessment involves the following in four dimensions: neuroclinical, hemodynamic, neurophysiologi$\mathrm{cal}$, and provocative. Neuroclinical evaluation, the cornerstone of the CRT, involves the continual monitoring of the patient for signs of cerebral ischemia for a period of approximately 20 minutes during which a nondetachable balloon is inflated in the parent artery and the patient is kept in the normotensive range. The most rudimentary hemodynamic evaluation involves simply observing the cross-flow of contrast material to the ipsilateral hemisphere via the communicating arteries as well as the lep- 
tomeningeal inflow and venous outflow in the involved territory. This, in our experience, has minimal value for predicting tolerance to parent artery sacrifice. A more useful hemodynamic assessment involves the aforementioned radionuclide CBF studies. Neurophysiological monitoring is accomplished very easily using EEG during the BTO. This adjunct confers another level of sensitivity to the evaluation in that, because it can potentially show changes due to ischemia in watershed zones, for instance, it can detect perfusion problems not evidenced by focal neurological deficits. The fourth dimension of the CRT as it is performed at our institution is provocative testing.

After patients undergo the CRT, we then stratify them into one of the following three categories based on their results: Group 1, passed in all four dimensions of testing; Group 2, passed neuroclinically but failed in another dimension; and Group 3, failed neuroclinically.

Group 1 patients do not undergo revascularization and their aneurysm may be treated using a Hunterian strategy or prolonged temporary occlusion if necessary. If a Hunterian procedure is required or prolonged temporary occlusion is necessary for clip-assisted reconstruction, Group 2 and 3 patients undergo a low-flow arterial bypass or a high-flow venous interpositional bypass procedure, respectively. An important point to note is that rarely is stratification into these groups cut and dry. Often times, patients who fall somewhere between two groups are placed into the group of criteria for which their CRT results most closely resemble, a concept that will be described in the proceeding case illustration.

\section{CASE ILLUSTRATION}

\section{Giant Supraclinoid Carotid Aneurysm}

This 49-year-old Asian woman without a significant medical history except for right-sided retroorbital pain was admitted to Northwestern Memorial Hospital with an incidentally discovered, partially thrombosed, giant supraclinoid CA aneurysm. On admission, the patient was alert and oriented in all three dimensions, and her neurological examination was unremarkable. Cerebral angiography demonstrated that the aneurysm imposed significant mass effect on both the $A_{1}$ and $M_{1}$ segments (Fig. 1). Our therapeutic plan was to attempt definitive exclusion of the lesion from the circulation by performing clip-assisted reconstruction with the understanding that, because of the complexity of the lesion and the presence of a hypoplastic efferent CA, a prolonged period of temporary CA occlusion or even intraoperative loss of the CA was possible. Cerebrovascular reserve testing showed very minimal left upper-extremity drift in conjunction with mild slowing and amplitude loss in the right anterior midtemporal leads on the EEG just after the balloon was inflated in the cervical right ICA. Both the clinical and neurophysiological deficits were transient and resolved in roughly 1 to 2 minutes. For the remaining 20 minutes of normotensive BTO, she was entirely neuroclinically intact and the EEG values were normal. When a hypotensive challenge was provoked, there were no signs of neurological impairment for the entire 5-minute period.

Because the BTO was essentially tolerated neuroclinically although there was minor evidence of transient im-

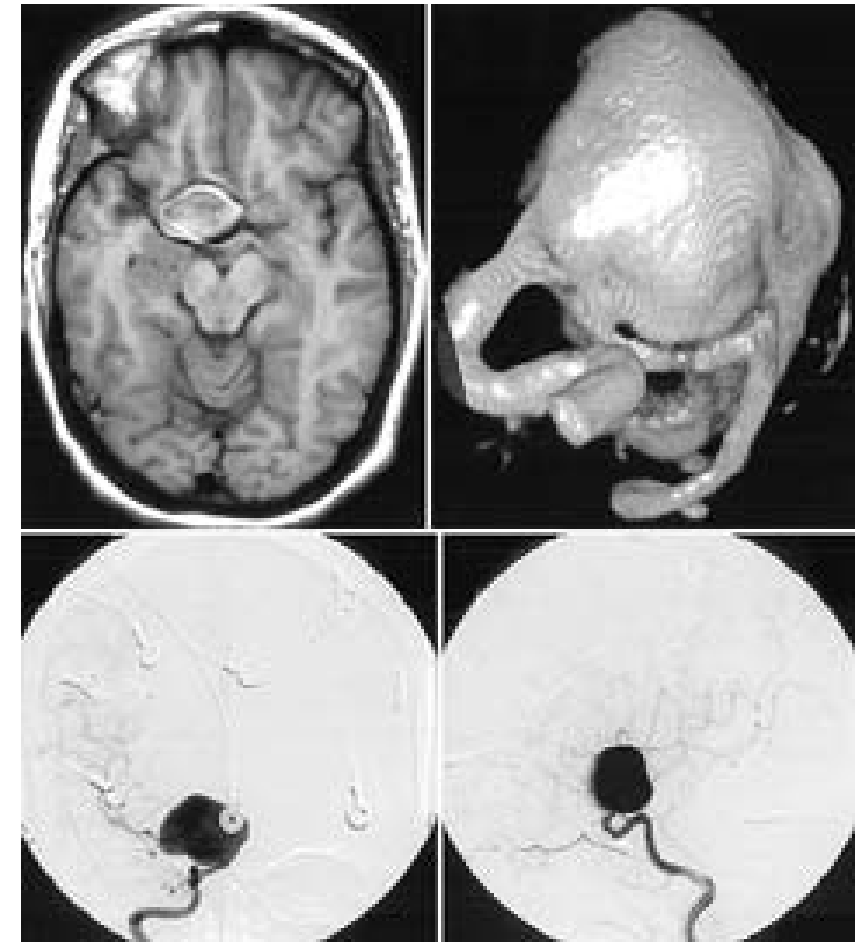

Fig. 1. Preoperative neurodiagnostic studies. Upper Left: Axial MR image of the lesion. Upper Right: A CT angiogram of the lesion demonstrating the complexity of its neck. Lower Left and Right: Anteroposterior (lower left) and lateral (lower right) cerebral angiograms obtained after right ICA injection, demonstrating a giant aneurysm arising from the supraclinoid CA.

pairment, the patient was considered to best belong to the Group 2 classification. As such, she was taken to the operating room at which point a superficial temporal arteryMCA bypass was performed. Immediately after patency of the low-flow bypass was verified angiographically (Fig. 2), clip-assisted reconstruction was conducted with the aid of retrograde suction decompression as described by Batjer and Samson. ${ }^{4}$

Routine intraoperative cerebral angiography demonstrated exclusion of the lesion from the intracranial circulation and satisfactory clip placement (Fig. 3). Of minor note, a transient third nerve palsy developed postoperatively. On routine postoperative cerebral angiography performed 5 days postoperatively, residual filling of the aneurysm sac was surprisingly demonstrated (Fig. 4). It was our assumption that a small amount of intraaneurysm thrombus was forced posteriorly by the clip closure and must have temporarily obliterated a tiny remaining neck deep to the blades. Ultimately, natural thrombolysis must have restored a channel of communication between the aneurysm remnant and the intracranial circulation. We considered endovascular coiling of the tiny remaining neck, but the sac had been widely opened for thrombus decompression at time of the first procedure, and we were concerned the coils might simply pass into the subarachnoid space. As a result, the patient was returned to the operating room at which point the clips were reapplied to the aneurysm neck to secure it and again exclude it from the circulation. A final postoperative cerebral angiogram 


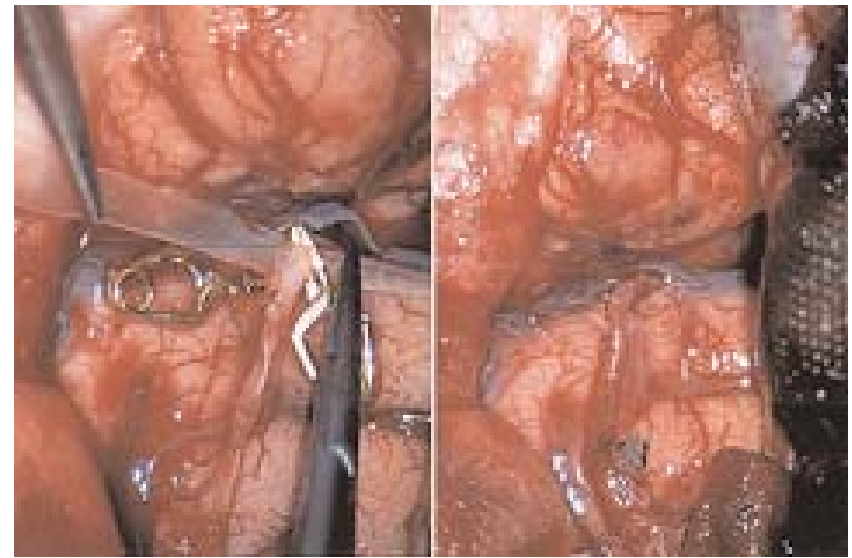

Fig. 2. Intraoperative surgical photographs demonstrating patency of the superficial temporal artery-MCA bypass with (left) and without (right) the temporary clips in place.

(Fig. 5) revealed complete exclusion of the aneurysm from the circulation with preservation of all afferent and efferent vasculature. She has made a full recovery.

\section{DISCUSSION}

Giant intracranial aneurysms, first defined by Morley and Barr $^{35}$ as being greater than $25 \mathrm{~mm}$ in maximum diameter, are uncommon clinical entities, although uniformly formidable from a technical standpoint. Excellent reviews on the natural history and epidemiology of these lesions have been published. ${ }^{1,3}$ Not dissimilar to their nongiant counterparts, giant aneurysms most frequently present clinically with either SAH, mass effect, or cerebral ischemia. Of note, giant aneurysms presenting with massive cerebral edema have also been reported. ${ }^{21}$ Several investigators have reported a dismal natural history of intradural giant aneurysms starting from the moment they become symptomatic in any manner, with most patients either dead or severely debilitated within only a few years. Because of this, some form of intervention is almost always warranted to cure or alter the natural history of this life-threatening condition.

The therapeutic approach to giant CA aneurysms is dichotomously divided into reconstructive and deconstructive strategies. Reconstructive strategies, accomplished by either clip placement or endovascular insertion of detachable coils, are preferred because they preserve cerebral hemodynamics. Because giant aneurysms frequently possess wide-based necks, endovascular aneurysmal thrombosis has produced less than optimal success rates. Deconstructive strategies, which principally involve surgical or endovascular Hunterian proximal arterial occlusion, are comparatively less desirable than direct clip-assisted reconstruction because they alter the postoperative cerebral hemodynamics and allow for potential new aneurysms to develop in the collateral system. Nevertheless, some giant aneurysms, either before or after intracranial exploration, prove unamenable to clip-assisted reconstruction and therefore must be, by default, treated by deconstructive measures.

Shortly after its introduction in 1967 by Yaşargil

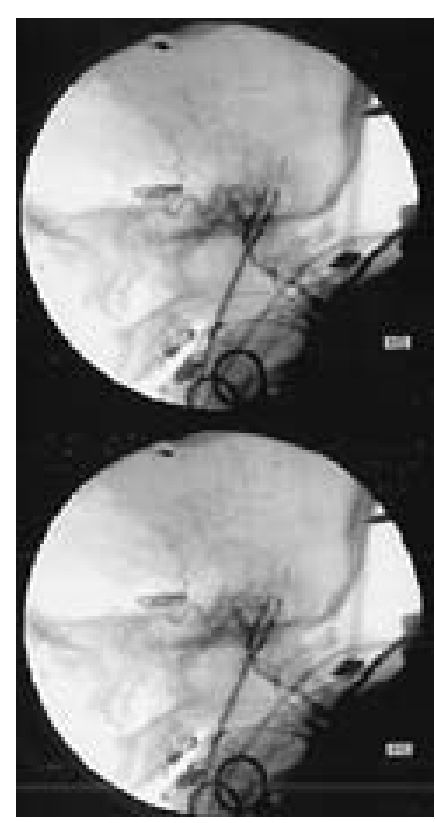

Fig. 3. Intraoperative right ICA angiogram obtained during the first operation, demonstrating patency of the right CA terminus with filling of MCA branches and clip exclusion of the aneurysm from the intracranial circulation.

alone ${ }^{50,51}$ and Donagh and Yaşargil, ${ }^{9}$ cerebral revascularization became extensively described in the neurosurgical literature as an adjunctive measure used in the treatment of complex aneurysms and cranial base tumors. $2,16,18,20,22,24$, 25,29,35,39-42,45 It has been traditionally undertaken using an EC-IC bypass; however, technically sophisticated highflow IC-IC venous interposition constructs have also been described. ${ }^{42}$ As previously described, in an effort to prevent cerebral ischemia when a Hunterian strategy is applied to treat a giant aneurysm or when prolonged temporary occlusion is anticipated, some neurovascular surgeons perform a prophylactic bypass in every patient, eliminating the necessity to determine cerebrovascular reserve capacity. The argument proposed by advocates of universal cerebral revascularization ${ }^{28}$ has focused on the delayed risk of cerebral ischemia, which they have asserted, is reduced by an EC-IC bypass following proximal parent arterial sacrifice. Additional reasons that universal revascularization is contented to be superior include a reported decreased rate of either de novo aneurysm formation or undetected aneurysm enlargement contralateral to a sacrificed CA and avoidance of the inherent risks associated with the BTO. By contrast, as proponents of a selective revascularization paradigm, we base our defense of this strategy on four central themes on which we will expound. 1) A small but significant inherent risk is associated with revascularization even in the best of hands. 2) Most cerebral ischemia following iatrogenic CA sacrifice is caused by embolic phenomena that have not been shown to be prevented or even abated after revascularization. 3) Both de novo aneurysm formation or existing aneurysm expansion, and even subsequent hemorrhage, may occur despite a bypass. 4) Balloon test occlusion-related risks have been exaggerated and, moreover, its predictive 


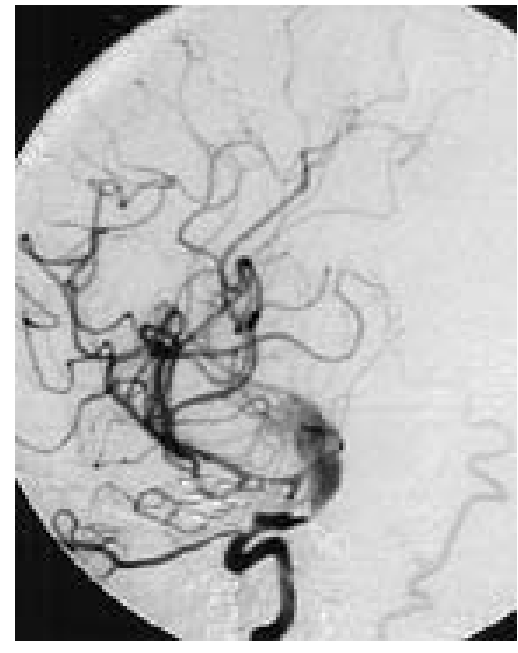

Fig. 4. Postoperative right ICA angiogram obtained after the first operation demonstrating residual filling of the aneurysm neck. Although there is smooth narrowing of the $A_{1}$ and $M_{1}$ segments due to mass effect from the aneurysm, it is considerably diminished compared with the preoperative studies.

value in patients with aneurysms in whom therapeutic CA sacrifice will be tolerated is quite good and is likely to improve in the future.

The first, and possibly most important reason to practice selective revascularization, is to avoid the potential complications associated with the procedure itself. Although not widely published in the neurosurgical literature, there are significant associated risks. In a consecutive series of 63 complex aneurysms in 61 patients published by Lawton and colleagues, ${ }^{28}$ in which all bypass procedures were performed by Dr. Spetzler, the authors reported a complication rate of $10 \%$; this evidence suggests significant procedure-related morbidity even in the best of hands. Of all the complications, $7 \%$ were attributed to the bypass per se and $3 \%$ to other nonneurological causes. Of note, in five patients in their series an epidural hematoma complicated their postoperative course. Its development was presumably related to the anticoagulation or antiplatelet therapy necessary to maintain patency of the graft. An additional point of contention related to surgery-induced complications is the diminished experience younger-generation neurosurgeons will have with performing the EC-IC bypass. Because of the unfavorable results reported by the EC/IC Study Group in $1985,{ }^{12}$ cerebral revascularization has been largely reserved for the treatment of complex aneurysms, trauma, and cranial base tumors. This considerable reduction in the number of ECIC bypasses performed by neurosurgeons today stands to reduce significantly the opportunity for the newer generation of neurovascular and cranial base surgeons to cultivate the technical skills needed to achieve excellent results. The overall complication rate of $10 \%$ reported by Lawton and colleagues, in light of this, should be considered a floor value, not an expected norm.

A second argument against universal revascularization relates to the incidence and cause of cerebral ischemic events following the use of Hunterian occlusion. Cerebral ischemia following parent artery sacrifice can be classified dichotomously with respect to time of occurrenceimmediate and delayed. A second, and more meaningful, manner by which to classify postocclusive ischemia is based on pathophysiological mechanism-thromboembolism compared with cerebral hypoperfusion. The earlier neurosurgical literature on CA occlusion in the treatment of intracranial aneurysms indicates that ischemic complications occurred in roughly $25 \%$ of patients. Of these, many were likely due to thromboembolic phenomena or cerebral vasospasm, an entity not widely recognized at the time. Certainly some cases of ischemia were due to a low-flow state that could be attributed to insufficient collateral circulation in the occluded arterial territory.

The authors of extensive reviews have determined that most ischemic events during or after therapeutic CA occlusion to promote aneurysmal thrombosis were thromboembolic in nature. For this reason, patients who, based on the CRT are classified as having Group 1 findings and

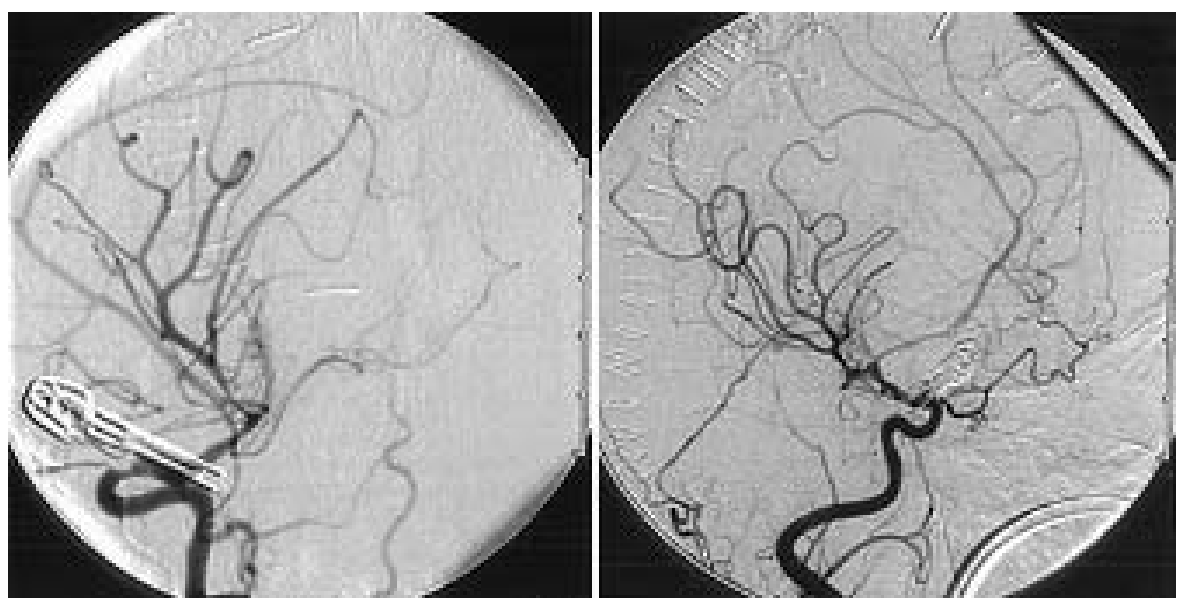

Fig. 5. Oblique (left) and lateral (right) right ICA angiograms obtained after the second operation at which time the clips were repositioned to exclude the aneurysm from the circulation. Of note, the $\mathrm{M}_{1}$ segment of the right MCA is severely stenotic and there is delayed transit time into the MCA territory, a finding consistent with cerebral vasospasm. 
should therefore tolerate CA occlusion due to their adequate collateral circulation, are still at risk of stroke because of distal embolization from the occluded CA segment. Despite this information, proponents of universal revascularization claim that an EC-IC bypass performed in patients undergoing parent artery sacrifice will significantly reduce the incidence of postocclusive stroke. Certainly a subset of postocclusive ischemia-that related to cerebral hypoperfusion in patients with marginal collateral circulation-will be alleviated by an EC-IC bypass that will augment CBF. Not only is there is no evidence to support a reduced incidence of ischemic stroke in patients with known adequate collateral circulation undergoing parent artery sacrifice and who have undergone revascularization, but because the principal mechanism of ischemia is thromboembolic there is no intuitive rationale either.

A study frequently quoted by advocates of universal revascularization is the retrospective review of 57 consecutive patients in whom intracranial aneurysms were treated with CA ligation. In this study Roski and colleagues, ${ }^{38}$ after a mean follow-up period of 12.5 years, reported an annual risk of ischemia-related neurological deficits, either transient or permanent, to be approximately $2.6 \%$. Significantly, 48 of the 57 patients presented with SAH. There is little doubt in our minds that many of these patients, reported to have suffered an ischemic event attributed to CA ligation, in fact, suffered vasospasm-induced cerebral ischemia, a major cause of delayed neurovascular insult which, at the time of this publication, was not widely appreciated by the neurosurgical community. Because the principal mechanism of postocclusive cerebral ischemia is distal thromboembolism due to intravascular CA "dead space" or the aneurysm itself, perioperative anticoagulation therapy, intracranial trapping, and endovascular release of detachable balloons at or proximal to the aneurysm neck ${ }^{8,15}$ are viable strategies by which to reduce the incidence of delayed stroke in patients undergoing a Hunterian occlusion and with confirmed adequate collateral circulation to sustain normal neuronal function.

A third point of contention in the debate between selective and universal revascularization relates to the incidence of de novo aneurysm formation, existing aneurysm expansion, and subsequent SAH in patients who have undergone CA sacrifice. ${ }^{6,27,30,32,49}$ Proponents of universal revascularization have asserted that the bypass-induced restoration of blood flow would minimize the hemodynamic changes occurring after parent artery sacrifice, which lead to de novo aneurysm formation and existing aneurysm expansion and rupture. Intuitively, there is no doubt that the greatly increased hemodynamic stress imposed on the contralateral CA tree after application of a Hunterian strategy to treat an aneurysm will yield to a higher incidence of aneurysm growth and subsequent rupture.

What makes us think then that ipsilateral cerebral revascularization will attenuate these risks? Certainly a highflow interposition saphenous vein graft causing reversal of flow in the MCA territory appears just as likely to cause the growth and rupture of aneurysms. In the two-stage treatment of a giant basilar caput aneurysm published by Heros and Ameri, ${ }^{20}$ Heros initially created an interpositional saphenous vein graft-assisted bypass to the $\mathrm{P}_{2}$ segment; the plan was to occlude the proximal basilar artery by using a Drake tourniquet the following day. Before the basilar artery could be sacrificed, the aneurysm ruptured and the patient suffered a massive, fatal aneurysmal SAH. This case clearly demonstrates that an EC-IC bypass can alter the cerebral hemodynamics in such a manner as to cause rupture of an aneurysm. One should also infer from this report that it is likely, because of the hemodynamic stress imposed by a bypass graft, that revascularization could also lead to aneurysm growth much in the same manner as CA occlusion in the absence of a bypass. Carotid artery occlusion simply imposes heightened hemodynamic stress on the contralateral circulation in an orthograde manner whereas the typical EC-IC bypass frequently results in increased flow in the ipsilateral circulation in a retrograde fashion.

Our fourth, and final, major reason for practicing selective revascularization is that it is our belief the aforementioned risk of the CRT, which is attributable to the BTO, has been exaggerated. Contemporary balloons are softer and less traumatic to tissue than earlier versions. Moreover, the predictive value of the BTO in patients in whom parent artery sacrifice will be to tolerated and who will not sustain an ischemic event due to cerebral hypoperfusion is, in our experience, quite good. In the large series of 300 patients who underwent BTO of the cerebrovascular reserve capacity published in 1991, Tarr and colleagues ${ }^{47}$ found that $3.7 \%$ of patients suffered a procedure-related complication, the most severe of which was stroke. In an even larger experience reported by Mathis and cowork$\mathrm{ers}^{31}$ the authors reviewed their experience performing 500 BTO procedures at the University of Pittsburgh between 1985 and 1993. The overall procedure-related complication rate was $3.2 \%$. Of note, however, there was a mere 1.2 and $0.4 \%$ incidence of transient and permanent neurological complications, respectively, due to the procedure. Although based solely on anecdotal evidence, it has been our institutional experience and that of others (Heros, RC: personal communication, 2003) that the BTO-related risk of complications is much lower, on the order of $1.5 \%$. Additionally, based on the favorable results of parent artery occlusion using an endovascular detachable balloon strategy, this technique is essentially associated with the same risk as the BTO.

With regard to the efficacy of the BTO in predicting tolerance to parent artery sacrifice in patients with complex $\mathrm{CA}$ aneurysms, Drake and colleagues ${ }^{11}$ reported ischemic complications in only three of their 114 patients in whom a Hunterian strategy-based procedure was performed in the absence of an adjunctive bypass to augment CBF, all of which were the result of a thromboembolic process. With the continual improvement of balloon-related technology and periocclusive pharmacology, it is our belief that both the risks associated with the BTO and endovascular Hunterian arterial occlusion will diminish. Equally impressive are the strides being made to improve the predictive value of a preoperative CRT. In their recently published retrospective account of 29 patients with large or giant CA aneurysms in whom therapeutic occlusion was contemplated, van Rooij and coworkers ${ }^{48}$ conducted, in addition to the BTO with both neuroclinical and neurophysiological monitoring, angiography to determine the presence or absence of synchronous venous filling of the two hemispheres to identify patients with adequate collat- 
eral circulation that would prevent low-flow ischemia. Of the 17 patients in whom evidence of neuroclinical, neurophysiological, and angiographic tolerance to CA occlusion was demonstrated, all then underwent endovascular CA sacrifice. In a mean follow-up period of 21 months, not one of these 17 patients experience a delayed cerebral ischemic event. One patient in whom the BTO was tolerated but asynchronous venous filling was visualized on angiography, experienced a delayed ipsilateral watershed infarction when CA sacrifice was performed urgently because of a rapidly expanding CA bifurcation aneurysm causing progressive compression of the optic apparatus.

\section{CONCLUSIONS}

Giant aneurysms of the anterior circulation are complex pathological entities that are both uncommon and technically challenging to treat. Therapy, either reconstructive or deconstructive, should be preceded by extensive testing of cerebrovascular reserve capacity. In patients known to be at risk of cerebral ischemia due to cerebral hypoperfusion, a revascularization procedure must be considered. The modalities by which tolerance to parent artery sacrifice are assessed, although currently imperfect, are improving. It should be understood, however, that these hemodynamically based studies cannot predict which patients will develop thromboembolic injuries after arterial sacrifice even when adequate collateral circulation exists. Nevertheless, it is our practice to undertake revascularization in patients in a selective, rather than universal, manner based on results of their cerebrovascular reserve assessment. It is our expectation that the tool of cerebral revascularization, a critical part of the armamentarium necessary to practice neurovascular surgery, will continue to play a dominant role in the management of complex cerebrovascular disease.

\section{References}

1. Anson JA: Epidemiology and natural history of giant aneurysms, in Awad IA, Barrow DL (eds): Giant Intracranial Aneurysms. Park Ridge: AANS, 1995, pp 23-34

2. Ausman JI, Pearce JE, de los Reyes RA, et al: Treatment of a high extracranial carotid artery aneurysm with CCA-MCA bypass and carotid ligation. Case report. J Neurosurg 58: 421-424, 1983

3. Barrow DL, Alleyne C: Natural history of giant intracranial aneurysms and indications for intervention. Clin Neurosurg 42: 214-244, 1995

4. Batjer HH, Samson DS: Retrograde suction decompression of giant paraclinoidal aneurysms. Technical note. J Neurosurg 73:305-306, 1990

5. Connors JJ III: Temporary test occlusion of the internal carotid artery, in Connors JJ III, Wojak JC (eds): Interventional Neuroradiology: Strategies and Practical Techniques. Philadelphia: WB Saunders, 1999, pp 377-389

6. Cuatico W, Cook AW, Tyshchenko V, et al: Massive enlargement of intracranial aneurysms following carotid ligation. Arch Neurol 17:609-613, 1967

7. de Vries EJ, Sekhar LN, Horton JA, et al: A new method to predict safe resection of the internal carotid artery. Laryngoscope 100:85-88, 1990

8. Debrun G, Fox A, Drake C, et al: Giant unclippable aneurysms: treatment with detachable balloons. AJNR 2:167-173, 1981

9. Donaghy RMP, Yaşargil MG (eds): Extracranial blood flow diversion, in Micro-Vascular Surgery: Report of First Conference, October 6-7, 1966, Mary Fletcher Hospital, Burlington, Vermont. St. Louis: Mosby, 1967

10. Drake CG: Giant intracranial aneurysms: experience with surgical treatment in 174 patients. Clin Neurosurg 26:12-95, 1979

11. Drake CG, Peerless SJ, Ferguson GG: Hunterian proximal arterial occlusion for giant aneurysms of the carotid circulation. $\mathbf{J}$ Neurosurg 81:656-665, 1994

12. EC/IC Bypass Study Group: Failure of extracranial-intracranial arterial bypass to reduce the risk of ischemic stroke. Results of an international randomized trial. N Engl J Med 313: 1191-1200, 1985

13. Eckard DA, Purdy PD, Bonte FJ: Temporary balloon occlusion of the carotid artery combined with brain blood flow imaging as a test to predict tolerance prior to permanent carotid sacrifice. AJNR 13:1565-1569, 1992

14. Erba SM, Horton JA, Latchaw RE, et al: Balloon test occlusion of the internal carotid artery with stable xenon/CT cerebral blood flow imaging. AJNR 9:533-538, 1988

15. Fox AJ, Viñuela F, Pelz DM, et al: Use of detachable balloons for proximal artery occlusion in the treatment of unclippable cerebral aneurysms. J Neurosurg 66:40-46, 1987

16. Gelber BR, Sundt TM Jr: Treatment of intracavernous and giant carotid aneurysms by combined internal carotid ligation and extra- to intracranial bypass. J Neurosurg 52:1-10, 1980

17. Gonzalez CF, Moret J: Balloon occlusion of the carotid artery prior to surgery for neck tumors. AJNR 11:649-652, 1990

18. Heros RC: Surgical management of unclippable intracranial aneurysms, in Schmidek HH (ed): Schmidek \& Sweet Operative Neurosurgical Techniques: Indications, Methods, and Results, ed 4. Philadelphia: WB Saunders, 2000, Vol 2, pp 1282-1295

19. Heros RC: Thromboembolic complications after combined internal carotid ligation and extra-to-intracranial bypass. Surg Neurol 21:75-79, 1984

20. Heros RC, Ameri AM: Rupture of a giant basilar aneurysm after saphenous vein interposition graft to the posterior cerebral artery. Case report. J Neurosurg 61:387-390, 1984

21. Heros RC, Kolluri S: Giant intracranial aneurysms presenting with massive cerebral edema. Neurosurgery 15:572-577, 1984

22. Heros RC, Nelson PB, Ojemann RG, et al: Large and giant paraclinoid aneurysms: surgical techniques, complications, and results. Neurosurgery 12:153-163, 1983

23. Horton JA, Jungreis CA, Pistoia F: Balloon test occlusion, in Sekhar LN, Janecka IP (eds): Surgery of Cranial Base Tumors. New York: Raven Press, 1993, pp 33-36

24. Hosobuchi Y: Giant intracranial aneurysms, in Wilkins RH, Rengachary SS (eds): Neurosurgery. New York: McGrawHill, 1985, pp 1404-1413

25. Jafar JJ, Russell SM, Woo HH: Treatment of giant intracranial aneurysms with saphenous vein extracranial-to-intracranial bypass grafting: indications, operative technique, and results in 29 patients. Neurosurgery 51:138-146, 2002

26. Jawad K, Miller D, Wyper DJ, et al: Measurement of CBF and carotid artery pressure compared with cerebral angiography in assessing collateral blood supply after carotid ligation. J Neurosurg 46:185-196, 1977

27. Klemme WM: Hemorrhage from a previously undemonstrated intracranial aneurysm as a late complication of carotid artery ligation. Case report. J Neurosurg 46:654-658, 1977

28. Lawton MT, Hamilton MG, Morcos JJ, et al: Revascularization and aneurysm surgery: current techniques, indications, and outcome. Neurosurgery 38:83-94, 1996

29. Linskey ME, Sekhar LN, Sen C: Cerebral revascularization in cranial base surgery, in Sekhar LN, Janecka IP (eds): Surgery of Cranial Base Tumors. New York: Raven Press, 1993, pp 45-68

30. Love JG, Dart LH: Results of carotid ligation with particular 
reference to intracranial aneurysms. J Neurosurg 27:89-93, 1967

31. Mathis JM, Barr JD, Jungreis CA, et al: Temporary balloon test occlusion of the internal carotid artery: experience in 500 cases. AJNR 16:749-754, 1995

32. Miller CA, Hill SA, Hunt WE: "De novo" aneurysms. A clinical review. Surg Neurol 24:173-180, 1985

33. Monsein LH, Jeffery PJ, van Heerden BB, et al: Assessing adequacy of collateral circulation during balloon test occlusion of the internal carotid artery with $99 \mathrm{mTc}-\mathrm{HMPAO}$ SPECT. AJNR 12:1045-1051, 1991

34. Morioka T, Matsushima T, Fujii K, et al: Balloon test occlusion of the internal carotid artery with monitoring of compressed spectral arrays (CSAs) of electroencephalogram. Acta Neurochir 101:29-34, 1989

35. Morley TP, Barr HW: Giant intracranial aneurysms: diagnosis, course, and management. Clin Neurosurg 16:73-94, 1969

36. Nishioka H: Report on the cooperative study of intracranial aneurysms and subarachnoid hemorrhage. Section VIII, part I: Results of the treatment of intracranial aneurysms by occlusion of the carotid artery in the neck. J Neurosurg 25:660-682, 1966

37. Peterman SB, Taylor A Jr, Hoffman JC Jr: Improved detection of cerebral hypoperfusion with internal carotid balloon test occlusion and 99mTc-HMPAO cerebral perfusion SPECT imaging. AJNR 12:1035-1041, 1991

38. Roski RA, Spetzler RF, Nulsen FE: Late complications of carotid ligation in the treatment of intracranial aneurysms. J Neurosurg 54:583-587, 1981

39. Sekhar LN, Bucur SD, Bank WO, et al: Venous and arterial bypass grafts for difficult tumors, aneurysms, and occlusive vascular lesions: evolution of surgical treatment and improved graft results. Neurosurgery 44:1207-1224, 1999

40. Sekhar LN, Duff JM, Kalavakonda C, et al: Cerebral revascularization using radial artery grafts for the treatment of complex intracranial aneurysms: techniques and outcomes for 17 patients. Neurosurgery 49:646-659, 2001

41. Spetzler RF, Carter LP: Revascularization and aneurysm surgery: current status. Neurosurgery 16:111-116, 1985

42. Spetzler RF, Fukushima T, Martin N, et al: Petrous carotidto-intradural carotid saphenous vein graft for intracavernous gi- ant aneurysm, tumor, and occlusive disease. J Neurosurg 73: 496-501, 1990

43. Standard SC, Ahuja A, Guterman LR, et al: Balloon test occlusion of the internal carotid artery with hypotensive challenge. AJNR 16:1453-1458, 1995

44. Steed DL, Webster MW, DeVries EJ, et al: Clinical observations on the effect of carotid artery occlusion on cerebral blood flow mapped by xenon computed tomography and its correlation with carotid artery back pressure. J Vasc Surg 11:38-44, 1990

45. Sundt TM Jr, Piepgras DG, Marsh WR, et al: Saphenous vein bypass grafts for giant aneurysms and intracranial occlusive disease. J Neurosurg 65:439-450, 1986

46. Swearingen B, Heros RC: Common carotid occlusion for unclippable carotid aneurysms: an old but still effective operation. Neurosurgery 21:288-295, 1987

47. Tarr RW, Jungreis CA, Horton JA, et al: Complications of preoperative balloon test occlusion of the carotid arteries: experience in 300 cases. Skull Base Surg 1:240-244, 1991

48. van Rooij WJ, Sluzewski M, Metz NH, et al: Carotid balloon occlusion for large and giant aneurysms: evaluation of a new test occlusion protocol. Neurosurgery 47:116-122, 2000

49. Winn HR, Richardson AE, Jane JA: Late morbidity and mortality of common carotid ligation for posterior communicating aneurysms. A comparison to conservative treatment. J Neurosurg 47:727-736, 1977

50. Yaşargil MG: Experimental small vessel surgery in the dog including patching and grafting of cerebral vessels and the formation of functional extra-intracranial shunts, in Donaghy RMP, Yaşargil MG (eds): Micro-Vascular Surgery: Report of First Conference, October 6-7, 1966, Mary Fletcher Hospital, Burlington, Vermont. St. Louis: Mosby, 1967

51. Yaşargil MG: Microsurgery Applied to Neurosurgery. Stuttgart: Thieme, 1969, pp 105-119

Manuscript received January 24, 2003.

Accepted in final form February 7, 2003.

Address reprint requests to: Brian A. O'Shaughnessy, M.D., 215

East Chicago Avenue \#1506, Chicago, Illinois 60611. email: branos@md.northwestern.edu. 